\title{
The general operator form for the total-momentum-dependent nucleon-nucleon potential
}

\author{
Kacper Topolnicki ${ }^{\mathrm{a}}$, Jacek Golak, Roman Skibiński, and Henryk Witała \\ M. Smoluchowski Institute of Physics, Jagiellonian University, PL-30348, Kraków, Poland
}

Received: 29 April 2016 / Revised: 16 June 2016

Published online: 19 July 2016

(c) The Author(s) 2016. This article is published with open access at Springerlink.com

Communicated by S. Hands

\begin{abstract}
In this paper we describe a procedure to obtain the general operator form of two-nucleon $(2 \mathrm{~N})$ potentials and apply it to the case of the $2 \mathrm{~N}$ potential that has an additional dependence on the total momentum of the system. This violates Galilean invariance but terms including the total momentum appear in some relativistic approaches. In operator form, the potential is expressed as a linear combination of a fixed number of known spin-momentum operators and scalar functions of momenta. Since the scalar functions effectively define the potentials, using the operator form significantly reduces the number of parameters that are needed in numerical implementations. The proposed operator form explicitly obeys the usual symmetries of rotational invariance, particle exchange, time reflection and parity.
\end{abstract}

\section{Symmetries of the general two-nucleon operator}

We consider quantum mechanical potential operators for a $2 \mathrm{~N}$ system in the non-relativistic domain. This, nonrelativistic, limitation is justified for few-nucleon forces, including the newest models derived from Chiral Effective Field Theory [1-4], since they are constructed to accurately describe the experimental data primarily at low energies. All our considerations will be performed in the momentum space with the momenta of the two individual particles 1, 2 in the initial (final) state denoted as $\boldsymbol{k}_{1}$, $\boldsymbol{k}_{2}\left(\boldsymbol{k}_{1}^{\prime}, \boldsymbol{k}_{2}^{\prime}\right)$. For the $2 \mathrm{~N}$ system the possible isospin structures are limited to the identity operator $\breve{1}$ (here and in the following we use the inverted hat " $\sim$ " to denote operators) and to the scalar product of the isospin operators $\check{\tau}(1) \cdot \check{\tau}(2)$. For this reason in the following it is sufficient to consider only the spin degrees of freedom, the extension to isospin is obvious. With this observation the momentum space projected operators can be represented using $4 \times 4$ matrices $(2 \times 2=4$ possible spin states for the $2 \mathrm{~N}$ system) and will be denoted, in the following, by using square brackets [...].

We focus on matrix elements of $2 \mathrm{~N}$ potentials $\check{V}$ of the form $\left\langle\boldsymbol{p}^{\prime} \boldsymbol{K}^{\prime}|\check{V}| \boldsymbol{p} \boldsymbol{K}\right\rangle$, where $\boldsymbol{p}^{\prime}=\frac{1}{2}\left(\boldsymbol{k}_{1}^{\prime}-\boldsymbol{k}_{2}^{\prime}\right)$, $\boldsymbol{p}=\frac{1}{2}\left(\boldsymbol{k}_{1}-\boldsymbol{k}_{2}\right)$ are the final and initial relative momenta of the two nucleons and $\boldsymbol{K}=\boldsymbol{k}_{1}+\boldsymbol{k}_{2}=\boldsymbol{k}_{1}^{\prime}+\boldsymbol{k}_{2}^{\prime}$ is the total momentum of the $2 \mathrm{~N}$ system. We allow the potential to be dependent on $\boldsymbol{K}$. This additional dependence can

\footnotetext{
${ }^{a}$ e-mail: kacper.topolnicki@uj.edu.pl
}

potentially break Galilean invariance, however terms of this type appear in non-relativistic reductions of relativistic expressions $[5,6]$. Our aim is to find the most general form of these matrix elements for operators that satisfy the usual symmetries. The motivation for this work is to reduce the numerical complexity of the so called "threedimensional" calculations [7-10] which directly utilize the "three-dimensional" momentum degrees of freedom of the nucleons without using partial wave decomposition. We also hope to extend our results to find the general form of the three-nucleon potential.

Momentum conservation means that

$$
\left\langle\boldsymbol{p}^{\prime} \boldsymbol{K}^{\prime}|\check{V}| \boldsymbol{p} \boldsymbol{K}\right\rangle \propto \delta^{3}\left(\boldsymbol{K}^{\prime}-\boldsymbol{K}\right) .
$$

More interesting is the rotational invariance since it involves both the momentum and spin degrees of freedom of the system. This symmetry will be considered in detail in the next sect. 2 .

We additionally assume the potential to be Hermitian and invariant under parity, time reversal and particle exchange. All these symmetry conditions are discussed in sect. 3. It should be noted that rotational invariance and discrete symmetries can be considered separately since the respective transformations commute with each other.

\section{Invariance under spatial rotations}

This section contains a description of an iterative algorithm to obtain the general rotationally invariant form of 
the $2 \mathrm{~N}$ potential. In the sections that follow additional symmetry constraints, mentioned above, will be added.

First we define the set $\mathbb{T}$ of all spin and momentum vector operators available for the construction of the $2 \mathrm{~N}$ potential

$$
\mathbb{T}=\left\{\check{\boldsymbol{\sigma}}(1), \check{\boldsymbol{\sigma}}(2), \check{\boldsymbol{p}}, \check{\boldsymbol{p}}^{\prime}, \check{\boldsymbol{K}}\right\},
$$

where, as mentioned earlier, $\boldsymbol{p}$ and $\boldsymbol{p}^{\prime}$ are the relative nucleon-nucleon momenta in the initial and final states respectively. $\boldsymbol{K}$ is the total (conserved) $2 \mathrm{~N}$ momentum and finally $\boldsymbol{\sigma}(1), \boldsymbol{\sigma}(2)$ are spin vectors (for particles 1,2 respectively). The inverted hat " $\sim$ " in (1) is used to indicate that the set $\mathbb{T}$ contains operators.

Operators which are invariant under spatial rotations can be constructed by combining various elements from $\mathbb{T}$ to form scalars. This could potentially lead to a great number of terms, since even lengthy scalar combinations are possible. Lengthy scalar combinations of vector operators can, however, be expanded by using simple vector identities to reveal a simple underlying structure. Considering, for example, the two following scalar combinations constructed from vector operators $\check{\boldsymbol{t}}_{i} \in \mathbb{T}(i$ is just the vector number and not a coordinate):

$$
\begin{aligned}
\left(\check{\boldsymbol{t}}_{1} \times\right. & \left.\left(\check{\boldsymbol{t}}_{2} \times \check{\boldsymbol{t}}_{3}\right)\right) \cdot\left(\check{\boldsymbol{t}}_{4} \times\left(\check{\boldsymbol{t}}_{5} \times\left(\check{\boldsymbol{t}}_{6} \times \check{\boldsymbol{t}}_{7}\right)\right)\right) \\
= & \left(\check{\boldsymbol{t}}_{1} \cdot \check{\boldsymbol{t}}_{3}\right)\left(\check{\boldsymbol{t}}_{2} \cdot \check{\boldsymbol{t}}_{5}\right)\left(\check{\boldsymbol{t}}_{4} \times \check{\boldsymbol{t}}_{6} \cdot \check{\boldsymbol{t}}_{7}\right) \\
& -\left(\check{\boldsymbol{t}}_{1} \cdot \check{\boldsymbol{t}}_{2}\right)\left(\check{\boldsymbol{t}}_{3} \cdot \check{\boldsymbol{t}}_{5}\right)\left(\check{\boldsymbol{t}}_{4} \times \check{\boldsymbol{t}}_{6} \cdot \check{\boldsymbol{t}}_{7}\right) \\
& +\left(\check{\boldsymbol{t}}_{1} \cdot \check{\boldsymbol{t}}_{2}\right)\left(\check{\boldsymbol{t}}_{4} \cdot \check{\boldsymbol{t}}_{5}\right)\left(\check{\boldsymbol{t}}_{3} \times \check{\boldsymbol{t}}_{6} \cdot \check{\boldsymbol{t}}_{7}\right) \\
& -\left(\check{\boldsymbol{t}}_{1} \cdot \check{\boldsymbol{t}}_{3}\right)\left(\check{\boldsymbol{t}}_{4} \cdot \check{\boldsymbol{t}}_{5}\right)\left(\check{\boldsymbol{t}}_{2} \times \check{\boldsymbol{t}}_{6} \cdot \check{\boldsymbol{t}}_{7}\right)
\end{aligned}
$$

and

$$
\begin{aligned}
&\left(\check{\boldsymbol{t}}_{1} \times \check{\boldsymbol{t}}_{2}\right) \cdot\left(\check{\boldsymbol{t}}_{3} \times\left(\check{\boldsymbol{t}}_{4} \times\left(\check{\boldsymbol{t}}_{5} \times \check{\boldsymbol{t}}_{6}\right)\right)\right) \\
&=\left(\check{\boldsymbol{t}}_{1} \cdot \check{\boldsymbol{t}}_{6}\right)\left(\check{\boldsymbol{t}}_{2} \cdot \check{\boldsymbol{t}}_{3}\right)\left(\check{\boldsymbol{t}}_{4} \cdot \check{\boldsymbol{t}}_{5}\right) \\
&-\left(\check{\boldsymbol{t}}_{1} \cdot \check{\boldsymbol{t}}_{3}\right)\left(\check{\boldsymbol{t}}_{2} \cdot \check{\boldsymbol{t}}_{6}\right)\left(\check{\boldsymbol{t}}_{4} \cdot \check{\boldsymbol{t}}_{5}\right) \\
&-\left(\check{\boldsymbol{t}}_{1} \cdot \check{\boldsymbol{t}}_{5}\right)\left(\check{\boldsymbol{t}}_{2} \cdot \check{\boldsymbol{t}}_{3}\right)\left(\check{\boldsymbol{t}}_{4} \cdot \check{\boldsymbol{t}}_{6}\right) \\
&+\left(\check{\boldsymbol{t}}_{1} \cdot \check{\boldsymbol{t}}_{3}\right)\left(\check{\boldsymbol{t}}_{2} \cdot \check{\boldsymbol{t}}_{5}\right)\left(\check{\boldsymbol{t}}_{4} \cdot \check{\boldsymbol{t}}_{6}\right),
\end{aligned}
$$

where we used

$$
\begin{aligned}
\boldsymbol{t}_{i} \times\left(\boldsymbol{t}_{j} \times \boldsymbol{t}_{k}\right) & =\boldsymbol{t}_{j}\left(\boldsymbol{t}_{i} \cdot \boldsymbol{t}_{\boldsymbol{k}}\right)-\boldsymbol{t}_{k}\left(\boldsymbol{t}_{i} \cdot \boldsymbol{t}_{\boldsymbol{j}}\right), \\
\left(\boldsymbol{t}_{i} \times \boldsymbol{t}_{j}\right) \times \boldsymbol{t}_{k} & =-\boldsymbol{t}_{i}\left(\boldsymbol{t}_{j} \cdot \boldsymbol{t}_{\boldsymbol{k}}\right)+\boldsymbol{t}_{j}\left(\boldsymbol{t}_{i} \cdot \boldsymbol{t}_{\boldsymbol{k}}\right), \\
\left(\boldsymbol{t}_{i} \times \boldsymbol{t}_{j}\right) \cdot\left(\boldsymbol{t}_{k} \times \boldsymbol{t}_{l}\right) & =\left(\boldsymbol{t}_{i} \cdot \boldsymbol{t}_{k}\right)\left(\boldsymbol{t}_{j} \cdot \boldsymbol{t}_{l}\right)-\left(\boldsymbol{t}_{i} \cdot \boldsymbol{t}_{l}\right)\left(\boldsymbol{t}_{j} \cdot \boldsymbol{t}_{k}\right),
\end{aligned}
$$

to perform the expansions on the right hand sides of (2) and $(3)$, it is easy to see that even very complicated scalar expressions can be built up from only two types of operators:

$$
\check{\boldsymbol{t}}_{i} \cdot \check{\boldsymbol{t}}_{j}, \quad\left(\check{\boldsymbol{t}}_{i} \times \check{\boldsymbol{t}}_{j}\right) \cdot \check{\boldsymbol{t}}_{k} .
$$

Thus, we define

$$
\mathbb{V}=\left\{\check{1}, \check{\boldsymbol{t}}_{i} \cdot \check{\boldsymbol{t}}_{j},\left(\check{\boldsymbol{t}}_{i} \times \check{\boldsymbol{t}}_{j}\right) \cdot \check{\boldsymbol{t}}_{k}\right\}
$$

to be a set of operators of type (4) that can be constructed from $\check{\boldsymbol{t}}_{i}, \check{\boldsymbol{t}}_{j}, \check{\boldsymbol{t}}_{k} \in \mathbb{T}$ and the identity operator $\check{1}$. The following steps describe the main algorithm leading to the general rotation-invariant form of the $2 \mathrm{~N}$ potential.
1) Construct the set $\mathbb{V}$. In order to keep the notation consistent in further iterations, let this first initial set of operators be denoted as $\overline{\mathbb{V}}_{0}=\mathbb{V}$.

2) The next step is the reduction of $\overline{\mathbb{V}}_{0}$ by eliminating those elements from $\overline{\mathbb{V}}_{0}$ that can be expressed as linear combinations of scalar functions of the momenta $\boldsymbol{p}, \boldsymbol{p}^{\prime}, \boldsymbol{K}$ and the remaining operators from $\overline{\mathbb{V}}_{0}$. This means that if operators $\check{X}, \check{Y}_{i} \in \overline{\mathbb{V}}_{0}$ and $\check{X} \neq \check{Y}_{i}$ and $\check{X}$ can be written as $\check{X}=\sum_{i=1}^{M-1} f_{i} \check{Y}_{i}$ with $f_{i}$ being a scalar function of momenta and $M$ being the number of elements in $\overline{\mathbb{V}}_{0}$, then $\check{X}$ can be eliminated.

3) After the elimination in step 2 we end up with a new, smaller set of operators $\mathbb{V}_{0}$ and, by its construction, it is clear that any operator from $\overline{\mathbb{V}}_{0}$ can be expressed by linear combinations of scalar functions of $\boldsymbol{p}, \boldsymbol{p}^{\prime}, \boldsymbol{K}$ and operators from $\mathbb{V}_{0}$. This set, $\mathbb{V}_{0}$, is the result of the first iteration of the algorithm. Additionally, this set will play a special role in constructing further iterations. In order to avoid confusion we give it an additional name, let $\mathbb{G}=\mathbb{V}_{0}$ be the generator.

4) Next we expand the set from the previous iteration. This is done by multiplying each operator from $\mathbb{V}_{0}$ by each operator from the generator $\mathbb{G}$. This larger set $\overrightarrow{\mathbb{V}}_{1}$ is then subjected to the same reduction procedure as in the first iteration (step 2). The resulting set $\mathbb{V}_{1}$ is the second iteration result.

5) These steps are repeated. We first expand the result of the previous $n$-th iteration $\mathbb{V}_{n-1}$ by multiplying each operator from this set by each operator from the generator $\mathbb{G}$. This larger set $\overline{\mathbb{V}}_{n}$ is then reduced (as in step 2 ) to produce $\mathbb{V}_{n}$, the $n+1$ iteration result.

6) The procedure is completed when new iterations do not introduce any new operators. The final result $\mathbb{V}_{N}$ can be shown to be composed from operators that can be used to construct the general, rotation-invariant, $2 \mathrm{~N}$ force.

For the $2 \mathrm{~N}$ case considered here, it turned out that this algorithm requires only two iterations. After the first iteration we end up with 11 operators in the generator $\mathbb{G}$ $\left(\mathbb{V}_{0}=\mathbb{G}\right)$ :

$$
\begin{aligned}
\check{G}_{1} & =\check{1}, \\
\check{G}_{2} & =\check{\boldsymbol{p}}^{\prime} \cdot \check{\boldsymbol{\sigma}}(1), \\
\check{G}_{3} & =\check{\boldsymbol{p}}^{\prime} \cdot \check{\boldsymbol{\sigma}}(2), \\
\check{G}_{4} & =\check{\boldsymbol{p}} \cdot \check{\boldsymbol{\sigma}}(1), \\
\check{G}_{5} & =\check{\boldsymbol{p}} \cdot \check{\boldsymbol{\sigma}}(2), \\
\check{G}_{6} & =\check{\boldsymbol{K}} \cdot \check{\boldsymbol{\sigma}}(1), \\
\check{G}_{7} & =\check{\boldsymbol{K}} \cdot \check{\boldsymbol{\sigma}}(2), \\
\check{G}_{8} & =\check{\boldsymbol{\sigma}}(1) \cdot \check{\boldsymbol{\sigma}}(2), \\
\check{G}_{9} & =\left(\check{\boldsymbol{p}}^{\prime} \times \check{\boldsymbol{\sigma}}(1)\right) \cdot \check{\boldsymbol{\sigma}}(2), \\
\check{G}_{10} & =(\check{\boldsymbol{p}} \times \check{\boldsymbol{\sigma}}(1)) \cdot \check{\boldsymbol{\sigma}}(2), \\
\check{G}_{11} & =(\check{\boldsymbol{K}} \times \check{\boldsymbol{\sigma}}(1)) \cdot \check{\boldsymbol{\sigma}}(2) .
\end{aligned}
$$

These operators are used to construct 16 operators in $\mathbb{V}_{1}=\left\{\check{O}_{i=1 \ldots 16}\right\}$ that will make up the general 
rotation-invariant form of the $2 \mathrm{~N}$ potential:

$$
\begin{aligned}
\check{O}_{1} & =\check{1}, \\
\check{O}_{2} & =\check{\boldsymbol{p}}^{\prime} \cdot \check{\boldsymbol{\sigma}}(1), \\
\check{O}_{3} & =\check{\boldsymbol{p}}^{\prime} \cdot \check{\boldsymbol{\sigma}}(2), \\
\check{O}_{4} & =\check{\boldsymbol{p}} \cdot \check{\boldsymbol{\sigma}}(1), \\
\check{O}_{5} & =\check{\boldsymbol{p}} \cdot \check{\boldsymbol{\sigma}}(2), \\
\check{O}_{6} & =\check{\boldsymbol{K}} \cdot \check{\boldsymbol{\sigma}}(1), \\
\check{O}_{7} & =\check{\boldsymbol{K}} \cdot \check{\boldsymbol{\sigma}}(2), \\
\check{O}_{8} & =\check{\boldsymbol{\sigma}}(1) \cdot \check{\boldsymbol{\sigma}}(2), \\
\check{O}_{9} & =\left(\check{\boldsymbol{p}}^{\prime} \times \check{\boldsymbol{\sigma}}(1)\right) \cdot \check{\boldsymbol{\sigma}}(2), \\
\check{O}_{10} & =(\check{\boldsymbol{p}} \times \check{\boldsymbol{\sigma}}(1)) \cdot \check{\boldsymbol{\sigma}}(2), \\
\check{O}_{11} & =(\check{\boldsymbol{K}} \times \check{\boldsymbol{\sigma}}(1)) \cdot \check{\boldsymbol{\sigma}}(2), \\
\check{O}_{12} & =\left(\check{\boldsymbol{p}}^{\prime} \cdot \check{\boldsymbol{\sigma}}(1)\right)(\check{\boldsymbol{p}} \cdot \check{\boldsymbol{\sigma}}(2)), \\
\check{O}_{13} & =\left(\check{\boldsymbol{p}}^{\prime} \cdot \check{\boldsymbol{\sigma}}(1)\right)(\check{\boldsymbol{p}} \cdot \check{\boldsymbol{\sigma}}(2)), \\
\check{O}_{14} & =\left(\check{\boldsymbol{p}}^{\prime} \cdot \check{\boldsymbol{\sigma}}(1)\right)(\check{\boldsymbol{K}} \cdot \check{\boldsymbol{\sigma}}(2)), \\
\check{O}_{15} & =(\check{\boldsymbol{p}} \cdot \check{\boldsymbol{\sigma}}(1))(\check{\boldsymbol{p}} \cdot \check{\boldsymbol{\sigma}}(2)), \\
\check{O}_{16} & =(\check{\boldsymbol{p}} \cdot \check{\boldsymbol{\sigma}}(1))(\check{\boldsymbol{K}} \cdot \check{\boldsymbol{\sigma}}(2)),
\end{aligned}
$$

It is clear that $\check{O}_{i=1 \ldots 11}=\check{G}_{i}$ and the second iteration introduced only 5 new operators $\check{O}_{i=12 \ldots 16}$. With our choice of elimination order, the third iteration does not contribute any new operators to the final set. By considering the way $\mathbb{V}_{1}$ was constructed, it can be shown that any rotation-invariant operator can be written as a linear combination of operators $\check{O}_{i} \in \mathbb{V}_{1}$ and scalar functions of the momenta $\alpha_{i}=\alpha_{i}\left(\boldsymbol{p}, \boldsymbol{p}^{\prime}, \boldsymbol{K}\right)$

$$
\check{V}=\alpha_{1} \check{O}_{1}+\ldots+\alpha_{16} \check{O}_{16} \text {. }
$$

In order to see this it is enough to consider only a single multiple product of operators constructed from elements of the form $\check{\boldsymbol{t}}_{i} \cdot \check{\boldsymbol{t}}_{j}$ and $\left(\check{\boldsymbol{t}}_{i} \times \check{\boldsymbol{t}}_{j}\right) \cdot \check{\boldsymbol{t}}_{k}$ since any scalar expression can be written as a linear combination of such products. For example, in expressions (2) and (3) we could consider each of the following products of operators separately:

$$
\begin{aligned}
& \left(\check{\boldsymbol{t}}_{1} \cdot \check{\boldsymbol{t}}_{3}\right)\left(\check{\boldsymbol{t}}_{2} \cdot \check{\boldsymbol{t}}_{5}\right)\left(\check{\boldsymbol{t}}_{4} \times \check{\boldsymbol{t}}_{6} \cdot \check{\boldsymbol{t}}_{7}\right), \\
& \left(\check{\boldsymbol{t}}_{1} \cdot \check{\boldsymbol{t}}_{2}\right)\left(\check{\boldsymbol{t}}_{3} \cdot \check{\boldsymbol{t}}_{5}\right)\left(\check{\boldsymbol{t}}_{4} \times \check{\boldsymbol{t}}_{6} \cdot \check{\boldsymbol{t}}_{7}\right), \\
& \left(\check{\boldsymbol{t}}_{1} \cdot \check{\boldsymbol{t}}_{2}\right)\left(\check{\boldsymbol{t}}_{4} \cdot \check{\boldsymbol{t}}_{5}\right)\left(\check{\boldsymbol{t}}_{3} \times \check{\boldsymbol{t}}_{6} \cdot \check{\boldsymbol{t}}_{7}\right), \\
& \left(\check{\boldsymbol{t}}_{1} \cdot \check{\boldsymbol{t}}_{3}\right)\left(\check{\boldsymbol{t}}_{4} \cdot \check{\boldsymbol{t}}_{5}\right)\left(\check{\boldsymbol{t}}_{2} \times \check{\boldsymbol{t}}_{6} \cdot \check{\boldsymbol{t}}_{7}\right), \\
& \left(\check{\boldsymbol{t}}_{1} \cdot \check{\boldsymbol{t}}_{6}\right)\left(\check{\boldsymbol{t}}_{2} \cdot \check{\boldsymbol{t}}_{3}\right)\left(\check{\boldsymbol{t}}_{4} \cdot \check{\boldsymbol{t}}_{5}\right), \\
& \left(\check{\boldsymbol{t}}_{1} \cdot \check{\boldsymbol{t}}_{3}\right)\left(\check{\boldsymbol{t}}_{2} \cdot \check{\boldsymbol{t}}_{6}\right)\left(\check{\boldsymbol{t}}_{4} \cdot \check{\boldsymbol{t}}_{5}\right), \\
& \left(\check{\boldsymbol{t}}_{1} \cdot \check{\boldsymbol{t}}_{5}\right)\left(\check{\boldsymbol{t}}_{2} \cdot \check{\boldsymbol{t}}_{3}\right)\left(\check{\boldsymbol{t}}_{4} \cdot \check{\boldsymbol{t}}_{6}\right), \\
& \left(\check{\boldsymbol{t}}_{1} \cdot \check{\boldsymbol{t}}_{3}\right)\left(\check{\boldsymbol{t}}_{2} \cdot \check{\boldsymbol{t}}_{5}\right)\left(\check{\boldsymbol{t}}_{4} \cdot \check{\boldsymbol{t}}_{6}\right) .
\end{aligned}
$$

We will now show how any multiple products of operators of this type, called a "chain" in the following, can be reduced to the general operator form (8). In appendix A we show an example illustrating the steps described in this paragraph when applied to a sample scalar $2 \mathrm{~N}$ operator. In general when looking at the first operator in a chain, there are only two mutually exclusive possibilities.
Either this operator is an element of $\mathbb{V}_{0}$ or it is not. If it is not then we can write it as a linear combination of operators from $\mathbb{V}_{0}$, this is guaranteed by the construction of $\mathbb{V}_{0}=\mathbb{G}$. Using this property we can write the whole chain as a linear combination and consider each of the new chains from the sum in this linear combination separately. We are guaranteed that each of these chains will start from an operator belonging to $\mathbb{V}_{0}$. Next, if a chosen chain has more than one operator, we can consider a subchain that contains the first pair of operators in a chain (otherwise we can stop since any elements of $\mathbb{V}_{0}$ can also be expressed by elements in $\mathbb{V}_{N}$, the final result of the algorithm). Looking this time at $\mathbb{V}_{1}$, again we have two possibilities. Either the considered sub-chain of operators is in $\mathbb{V}_{1}$ or it is not. If it is not then it must be a linear combination of operators in $\mathbb{V}_{1}$, this is guaranteed by the construction of $\mathbb{V}_{1}$. Similarly, we can use this property to write the whole chain as a linear combination. This results in a sum containing a certain number of chains with the guarantee that each chain will begin with an operator from $\mathbb{V}_{1}$. Again, each chain can be considered separately and the same steps as above can be repeated. We look at a sub-chain that contains the next operator (if there is one) and create chains that start with operators from $\mathbb{V}_{2}$. Next we look at a sub-chain that contains yet another operator (if there is one) and create chains that start with operators from $\mathbb{V}_{3}$, etc. After each repetition, we will end up with chains, each starting with an operator from $\mathbb{V}_{2}$, $\mathbb{V}_{3}, \ldots$ After we reach $\mathbb{V}_{N}$ (the final result of the algorithm, for our $2 \mathrm{~N}$ case $\mathbb{V}_{N}=\mathbb{V}_{1}$ ) there is no need to look at $\mathbb{V}_{N+1}$. We can continue to consider larger sub-chains and in each case look at $\mathbb{V}_{N}$. The procedure stops if there are no new operators to add to a sub-chain. Finally we will end up with a linear combination of chains, each containing a single element from $\mathbb{V}_{N}$, very similar to the sum (8).

Using the same arguments, it is also easy to see that $\mathbb{V}_{N}$ is complete. Any product of operators from $\mathbb{V}_{N}$ can be written as a linear combination of operators from $\mathbb{V}_{N}$ and scalar functions of the momenta. This can be immediately verified by considering the procedure from the previous paragraph.

Now we describe a more practical aspect of the implementation. It is important to make sure that the reduction from steps 2 and 4 of the operator-generating procedure is implemented in an efficient way since this is the most demanding part of the algorithm. In our calculations we use a numerical approach to perform the reduction. We take all operators contained in $\overline{\mathbb{V}}_{n-1}$ at the beginning of the $n$-th iteration and consider each operator as a matrix element in momentum space and as an operator in spin space. Next, since there are 4 possible spin states, we create the $4 \times 4$ matrix representation for each operator. We then choose random numerical values for the momentum vectors $\boldsymbol{p}, \boldsymbol{p}^{\prime}, \boldsymbol{K}$ and substitute these in the matrix representation. At this point, we have a set of matrices that are composed of complex numbers. Checking if an operator can be expressed as a linear combination of the other operators multiplied by scalar functions of momenta now becomes an easy task. Namely, one particular matrix is taken out of the set $\overline{\mathbb{V}}_{n-1}$ and written as a vector in its 
natural basis

$$
\left(\begin{array}{ccc}
1 & 0 & \ldots \\
0 & 0 & \ldots \\
\vdots & \vdots & \ddots
\end{array}\right)^{4 \times 4},\left(\begin{array}{ccc}
0 & 1 & \ldots \\
0 & 0 & \ldots \\
\vdots & \vdots & \ddots
\end{array}\right)^{4 \times 4}, \ldots
$$

so that the vectors elements are simply appropriate components of the matrix representation. Let this 16dimensional vector be $b$. The remaining operators from the set are also written in the same way in this basis. These vectors can be stacked together to create a $(M-1) \times 16$ matrix $A$, where $M$ is the number of operators in $\overline{\mathbb{V}}_{n-1}$. All that remains is to solve a linear equation $A x=b$ and the results $x$ are the values of the $M-1$ scalar functions with the momenta $\boldsymbol{p}, \boldsymbol{p}^{\prime}, \boldsymbol{K}$ having the appropriate randomly substituted values. To be on the safe side, multiple different momentum substitutions can be used. If no numerical solution to $A x=b$ can be found, then the operator is considered independent. If a solution can be found, then the operator is left out from $\overline{\mathbb{V}}_{n-1}$.

Caution is advised however, since it is tempting to use, instead of steps 1-6 of the operator generating algorithm, a procedure similar to Gauss elimination: stack the $M$ vectors to create a $M \times 16$ matrix, then multiply the rows by numbers and add rows to each other in order to set as many rows as possible to 0 . This procedure will lead to errors since we can only multiply each operator by a scalar function. A number might violate this requirement (e.g. we cannot multiply by the $z$ component of $\boldsymbol{p}$ - since it is clearly not a scalar function).

The procedure described in this section can also be applied to systems of three or more particles. Only slight modifications are required. For instance when dealing with the three-nucleon force in momentum space, the potential could be constructed from the Jacobi momenta in the final and initial states $\boldsymbol{p}^{\prime}, \boldsymbol{q}^{\prime}, \boldsymbol{p}, \boldsymbol{q}$ and the total momentum $\boldsymbol{K}$. This together with the addition of the third spin operator $\check{\boldsymbol{\sigma}}(3)$ leads to a different form of the set (1) that can be used to construct the general form of the spatial rotationinvariant three-nucleon potential

$$
\mathbb{T}=\left\{\check{\boldsymbol{\sigma}}(1), \check{\boldsymbol{\sigma}}(2), \check{\boldsymbol{\sigma}}(3), \check{\boldsymbol{p}}, \check{\boldsymbol{q}}, \check{\boldsymbol{p}}^{\prime}, \check{\boldsymbol{q}}^{\prime}, \check{\boldsymbol{K}}\right\}
$$

With this in mind, the procedure can be carried out with almost no modifications.

\section{Additional symmetries}

At this point we have at our disposal 16 operators $\check{O}_{i}$ from (7) that make up the rotationally invariant $2 \mathrm{~N}$ potential. In addition to this we require that the potential be Hermitian and symmetric with respect to parity, time reversal and particle exchange. We will consider a group of transformations composed from the identity operator $\check{1}$, spatial reflections $\check{T}^{\mathrm{SR}}$, time reflections $\check{T}^{\mathrm{TR}}$, Hermitian conjugations $\check{T}^{\mathrm{HR}}\left(\check{X}^{\dagger}=\check{T}^{\mathrm{HR}} \check{X}\right)$ and particle exchange $\check{T}^{\mathrm{PE}}$. We require the potential to be symmetric with respect to this group. The explicit set of discrete operations that form the group will be denoted by $\mathbb{D}$ :

$$
\mathbb{D}=\left\{\check{1}, \check{T}^{\mathrm{SR}}\right\} \times\left\{\check{1}, \check{T}^{\mathrm{TR}}\right\} \times\left\{\check{1}, \check{T}^{\mathrm{HR}}\right\} \times\left\{\check{1}, \check{T}^{\mathrm{PE}}\right\}
$$

and this set is a direct product of 4 simple groups. In practice we use the $4 \times 4$ matrix representation of operators to implement transformations acting on operator $\check{O}\left(\boldsymbol{p}^{\prime}, \boldsymbol{p}, \boldsymbol{K}\right)$. Time reflection $\check{T}^{\mathrm{TR}}$ was implemented using

$$
\begin{aligned}
& {\left[\check{O}\left(\boldsymbol{p}^{\prime}, \boldsymbol{p}, \boldsymbol{K}\right)\right] \rightarrow} \\
& {\left[i \sigma_{y} \otimes i \sigma_{y}\right]\left[\check{O}\left(-\boldsymbol{p},-\boldsymbol{p}^{\prime},-\boldsymbol{K}\right)\right]\left[i \sigma_{y} \otimes i \sigma_{y}\right]^{-1},}
\end{aligned}
$$

where $\sigma_{y}$ is the Pauli matrix and $\otimes$ denotes the Kronecker product. Particle exchange $\check{T}^{\mathrm{PE}}$ was implemented using

$$
\left[\check{O}\left(\boldsymbol{p}^{\prime}, \boldsymbol{p}, \boldsymbol{K}\right)\right] \rightarrow\left[\check{P}_{12}\right]\left[\check{O}\left(-\boldsymbol{p}^{\prime},-\boldsymbol{p}, \boldsymbol{K}\right)\right]\left[\check{P}_{12}\right]
$$

where $\left[\check{P}_{12}\right]$ is a $4 \times 4$ matrix implementing particle permutations in the spin space. Spatial reflections $\check{T}^{\mathrm{SR}}$ were implemented using

$$
\left[\check{O}\left(\boldsymbol{p}^{\prime}, \boldsymbol{p}, \boldsymbol{K}\right)\right] \rightarrow\left[\check{O}\left(-\boldsymbol{p}^{\prime},-\boldsymbol{p},-\boldsymbol{K}\right)\right] .
$$

Finally the Hermitian conjugate $\check{T}^{\mathrm{HR}}$

$$
\left[\check{O}\left(\boldsymbol{p}^{\prime}, \boldsymbol{p}, \boldsymbol{K}\right)\right] \rightarrow\left[\check{O}\left(\boldsymbol{p}, \boldsymbol{p}^{\prime}, \boldsymbol{K}\right)\right]^{\dagger}
$$

Parallel to the matrix implementation of the symmetry transformations we used the symbolic equivalents of the same transformations where we work directly with analytical expressions involving $\boldsymbol{p}^{\prime}, \boldsymbol{p}, \boldsymbol{K}, \boldsymbol{\sigma}(1), \boldsymbol{\sigma}(2)$.

In order to add the additional symmetries, a symmetrization procedure is applied to the general form of the potential (8)

$$
\check{V} \rightarrow \sum_{\check{T} \in \mathbb{D}} \check{T} \check{V}
$$

where the sum is over all transformations of the group $\mathbb{D}$. After this transformation we are guaranteed that the resulting operator is symmetric with respect to all transformations from (9). This can be immediately verified by applying any transformation $\check{D} \in \mathbb{D}$ to the new operator:

$$
\check{D} \sum_{\check{T} \in \mathbb{D}} \check{T} \check{V}=\sum_{\check{T} \in \mathbb{D}} \check{D} \check{T} \check{V}=\sum_{\check{T} \in \mathbb{D}} \check{T} \check{V} .
$$

This equation is satisfied since $\mathbb{D}$ is a group and $\check{D} \check{T}$ is just another element of $\mathbb{D}$. The combination of the two operators only changes the order of the summation on the right hand side of (15).

When applying the procedure (14) to (8), we can focus on each element of the sum separately. It is important, however, to keep in mind that the procedure is applied to the scalar functions of momenta and to the operators at the same time:

$$
\sum_{\breve{T} \in \mathbb{D}} \check{T} \check{V}=\sum_{\check{T} \in \mathbb{D}} \check{T}\left(\alpha_{1} \check{O}_{1}+\ldots+\alpha_{16} \check{O}_{16}\right) .
$$

For this reason it is important to consider the number and type of arguments for the scalar function. A simple 
calculation reveals that for the $2 \mathrm{~N}$ system with 3 vectors, the scalar function can have in general $3 \times 3-3=6$ arguments (number of vectors $\times$ number of dimensions - number of constraints). It is easy to construct 6 scalar combinations from $\boldsymbol{p}, \boldsymbol{p}^{\prime}$ and $\boldsymbol{K}$ :

$\alpha\left(\boldsymbol{p}^{\prime}, \boldsymbol{p}, \boldsymbol{K}\right)=\alpha\left(\boldsymbol{p}^{\prime} \cdot \boldsymbol{p}^{\prime}, \boldsymbol{p} \cdot \boldsymbol{p}, \boldsymbol{K} \cdot \boldsymbol{K}, \boldsymbol{p}^{\prime} \cdot \boldsymbol{p}, \boldsymbol{p} \cdot \boldsymbol{K}, \boldsymbol{K} \cdot \boldsymbol{p}^{\prime}\right)$,

but such a simple choice of arguments is incorrect and can be misleading. To understand this we can consider two momentum transformations

$$
p \rightarrow-p, \quad p^{\prime} \rightarrow-p^{\prime}
$$

and

$$
\boldsymbol{K} \rightarrow-\boldsymbol{K}
$$

They are a part of parity, time reversal and particle exchange in (10), (11), (12), (13). It turns out that they lead to the same function

$$
\alpha\left(-\boldsymbol{p}^{\prime},-\boldsymbol{p}, \boldsymbol{K}\right)=\alpha\left(\boldsymbol{p}^{\prime}, \boldsymbol{p},-\boldsymbol{K}\right) .
$$

If arguments containing a triple product

$$
p \times p^{\prime} \cdot K
$$

are included and

$$
\begin{aligned}
& \alpha\left(\boldsymbol{p}^{\prime}, \boldsymbol{p}, \boldsymbol{K}\right)= \\
& \alpha\left(\boldsymbol{p}^{\prime} \cdot \boldsymbol{p}^{\prime}, \boldsymbol{p} \cdot \boldsymbol{p}, \boldsymbol{K} \cdot \boldsymbol{K}, \boldsymbol{p}^{\prime} \cdot \boldsymbol{p}, \boldsymbol{p} \cdot \boldsymbol{K}, \boldsymbol{p} \times \boldsymbol{p}^{\prime} \cdot \boldsymbol{K}\right),
\end{aligned}
$$

then the same transformations lead to different functions. In practice the procedure (14) was carried out using symbolic programming within the Mathematica ${ }^{\circledR}[11]$ package and we used (17) as test functions.

Another issue to consider is the type of the scalar functions. We considered the properties of the scalar functions $\alpha$ under time reversal, particle exchange and Hermitian conjugation. After determining the group of transformations that change the arguments of the scalar functions we chose a representation for $\alpha$

$$
\begin{aligned}
\alpha= & \beta^{-1,-1,-1}+\beta^{-1,-1,1}+\beta^{-1,1,-1} \\
& +\beta^{-1,1,1}+\beta^{1,-1,-1}+\beta^{1,-1,1}+\beta^{1,1,-1}+\beta^{1,1,1}
\end{aligned}
$$

where $\beta^{n_{1}, n_{2}, n_{3}}$ is any scalar function that changes its sign to $n_{1}, n_{2}$ and $n_{3}$ under the transformations

$$
\begin{aligned}
& \beta^{n_{1}, n_{2}, n_{3}}\left(\boldsymbol{p}^{\prime}, \boldsymbol{p}, \boldsymbol{K}\right)=n_{1} \beta^{n_{1}, n_{2}, n_{3}}\left(-\boldsymbol{p},-\boldsymbol{p}^{\prime}, \boldsymbol{K}\right), \\
& \beta^{n_{1}, n_{2}, n_{3}}\left(\boldsymbol{p}^{\prime}, \boldsymbol{p}, \boldsymbol{K}\right)=n_{2} \beta^{n_{1}, n_{2}, n_{3}}\left(\boldsymbol{p}^{\prime}, \boldsymbol{p},-\boldsymbol{K}\right), \\
& \beta^{n_{1}, n_{2}, n_{3}}\left(\boldsymbol{p}^{\prime}, \boldsymbol{p}, \boldsymbol{K}\right)=n_{3} \beta^{n_{1}, n_{2}, n_{3}}\left(-\boldsymbol{p}^{\prime},-\boldsymbol{p}, \boldsymbol{K}\right) .
\end{aligned}
$$

Considering the purely real and purely imaginary scalar functions of different types (18), after performing the symmetrization procedure (14), we end up with a large set of operators. Next we use a reduction procedure described in sect. 2 but with additional tests for the scalar function type $\beta^{n_{1}, n_{2}, n_{3}}$. This eliminates those operators that can be expressed by a linear combination of the
Table 1. The $n_{1}(i), n_{2}(i), n_{3}(i)$ values for $\beta_{i}^{n_{1}(i), n_{2}(i), n_{3}(i)}$ from $(20)$.

\begin{tabular}{c|rrr}
\hline$i$ & $n_{1}(i)$ & $n_{2}(i)$ & $n_{3}(i)$ \\
\hline 1 & -1 & -1 & -1 \\
2 & -1 & 1 & 1 \\
3 & 1 & -1 & -1 \\
4 & 1 & -1 & -1 \\
5 & 1 & -1 & 1 \\
6 & 1 & 1 & 1 \\
7 & 1 & 1 & 1 \\
8 & 1 & 1 & 1 \\
9 & 1 & 1 & 1 \\
10 & 1 & 1 & 1 \\
11 & -1 & -1 & 1 \\
12 & -1 & -1 & 1 \\
13 & -1 & 1 & -1 \\
14 & 1 & -1 & 1 \\
15 & 1 & 1 & -1 \\
16 & 1 & 1 & -1 \\
\hline
\end{tabular}

remaining operators and scalar functions (of appropriate type).

We end up with 16 independent operators

$$
\begin{aligned}
\check{W}_{1}= & (\boldsymbol{p} \cdot \check{\boldsymbol{\sigma}}(1))(\boldsymbol{K} \cdot \check{\boldsymbol{\sigma}}(2))+(\boldsymbol{p} \cdot \check{\boldsymbol{\sigma}}(2))(\boldsymbol{K} \cdot \check{\boldsymbol{\sigma}}(1)) \\
& +(\boldsymbol{K} \cdot \check{\boldsymbol{\sigma}}(1))\left(\boldsymbol{p}^{\prime} \cdot \check{\boldsymbol{\sigma}}(2)\right)+(\boldsymbol{K} \cdot \check{\boldsymbol{\sigma}}(2))\left(\boldsymbol{p}^{\prime} \cdot \check{\boldsymbol{\sigma}}(1)\right), \\
\check{W}_{2}= & (\boldsymbol{p} \cdot \check{\boldsymbol{\sigma}}(1))(\boldsymbol{p} \cdot \check{\boldsymbol{\sigma}}(2))-\left(\boldsymbol{p}^{\prime} \cdot \check{\boldsymbol{\sigma}}(2)\right)\left(\boldsymbol{p}^{\prime} \cdot \check{\boldsymbol{\sigma}}(1)\right), \\
\check{W}_{3}= & (\boldsymbol{p} \cdot \check{\boldsymbol{\sigma}}(2))\left(\boldsymbol{p}^{\prime} \cdot \check{\boldsymbol{\sigma}}(1)\right)-(\boldsymbol{p} \cdot \check{\boldsymbol{\sigma}}(1))\left(\boldsymbol{p}^{\prime} \cdot \check{\boldsymbol{\sigma}}(2)\right), \\
\check{W}_{4}= & (\boldsymbol{p} \cdot \check{\boldsymbol{\sigma}}(1))(\boldsymbol{K} \cdot \check{\boldsymbol{\sigma}}(2))+(\boldsymbol{p} \cdot \check{\boldsymbol{\sigma}}(2))(\boldsymbol{K} \cdot \check{\boldsymbol{\sigma}}(1)) \\
& -(\boldsymbol{K} \cdot \check{\boldsymbol{\sigma}}(1))\left(\boldsymbol{p}^{\prime} \cdot \check{\boldsymbol{\sigma}}(2)\right)-(\boldsymbol{K} \cdot \check{\boldsymbol{\sigma}}(2))\left(\boldsymbol{p}^{\prime} \cdot \check{\boldsymbol{\sigma}}(1)\right), \\
\check{W}_{5}= & \left(\boldsymbol{p}+\boldsymbol{p}^{\prime}\right) \cdot \check{\boldsymbol{\sigma}}(1) \times \check{\boldsymbol{\sigma}}(2), \\
\check{W}_{6}= & \check{1} \\
\check{W}_{7}= & \check{\boldsymbol{\sigma}}(1) \cdot \check{\boldsymbol{\sigma}}(2), \\
\check{W}_{8}= & (\boldsymbol{p} \cdot \check{\boldsymbol{\sigma}}(1))\left(\boldsymbol{p}^{\prime} \cdot \check{\boldsymbol{\sigma}}(2)\right)+(\boldsymbol{p} \cdot \check{\boldsymbol{\sigma}}(2))\left(\boldsymbol{p}^{\prime} \cdot \check{\boldsymbol{\sigma}}(1)\right), \\
\check{W}_{9}= & (\boldsymbol{p} \cdot \check{\boldsymbol{\sigma}}(1))(\boldsymbol{p} \cdot \check{\boldsymbol{\sigma}}(2))+\left(\boldsymbol{p}^{\prime} \cdot \check{\boldsymbol{\sigma}}(1)\right)\left(\boldsymbol{p}^{\prime} \cdot \check{\boldsymbol{\sigma}}(2)\right), \\
\check{W}_{10}= & (\boldsymbol{p} \cdot \check{\boldsymbol{\sigma}}(1))(\boldsymbol{K} \cdot \check{\boldsymbol{\sigma}}(2))-(\boldsymbol{p} \cdot \check{\boldsymbol{\sigma}}(2))(\boldsymbol{K} \cdot \check{\boldsymbol{\sigma}}(1)) \\
& -(\boldsymbol{K} \cdot \check{\boldsymbol{\sigma}}(1))\left(\boldsymbol{p}^{\prime} \cdot \check{\boldsymbol{\sigma}}(2)\right)+(\boldsymbol{K} \cdot \check{\boldsymbol{\sigma}}(2))\left(\boldsymbol{p}^{\prime} \cdot \check{\boldsymbol{\sigma}}(1)\right), \\
\check{W}_{11}= & i\left(\boldsymbol{p} \cdot(\check{\boldsymbol{\sigma}}(1)-\check{\boldsymbol{\sigma}}(2))+\boldsymbol{p}^{\prime} \cdot(\check{\boldsymbol{\sigma}}(1)-\check{\boldsymbol{\sigma}}(2))\right), \\
\check{W}_{12}= & i \boldsymbol{K} \cdot(\check{\boldsymbol{\sigma}}(1)+\check{\boldsymbol{\sigma}}(2)), \\
\check{W}_{13}= & i\left(\boldsymbol{p} \cdot(\check{\boldsymbol{\sigma}}(1)+\check{\boldsymbol{\sigma}}(2))-\boldsymbol{p}^{\prime} \cdot(\check{\boldsymbol{\sigma}}(1)+\check{\boldsymbol{\sigma}}(2))\right), \\
\check{W}_{14}= & i\left(\boldsymbol{p} \cdot(\check{\boldsymbol{\sigma}}(1)-\check{\boldsymbol{\sigma}}(2))-\boldsymbol{p}^{\prime} \cdot(\check{\boldsymbol{\sigma}}(1)-\check{\boldsymbol{\sigma}}(2))\right), \\
\check{W}_{15}= & i\left(\boldsymbol{p} \cdot(\check{\boldsymbol{\sigma}}(1)+\check{\boldsymbol{\sigma}}(2))+\boldsymbol{p}^{\prime} \cdot(\check{\boldsymbol{\sigma}}(1)+\check{\boldsymbol{\sigma}}(2))\right), \\
\check{W}_{16}= & i \boldsymbol{K} \cdot(\check{\boldsymbol{\sigma}}(2)-\check{\boldsymbol{\sigma}}(1)),
\end{aligned}
$$

that make up the general operator form of the $2 \mathrm{~N}$ potential

$$
\check{V}=\sum_{i=1}^{16} \beta_{i}^{n_{1}(i), n_{2}(i), n_{3}(i)} \check{W}_{i} .
$$

The numbers $n_{1}, n_{2}, n_{3}$ assigned to each real scalar function are given in table 1. Equation (20) is our final expression for the $2 \mathrm{~N}$ potential dependent on the initial 
and final relative momentum and the total momentum of the system. This form is Hermitian and constructed to implicitly obey rotation symmetry as well as parity, time reversal and particle exchange. It can be additionaly checked that removing the Hermiticity condition changes (20) by allowing the functions $\beta$ to be complex. In principle, a very similar procedure can be used to construct the general operator form of the $3 \mathrm{~N}$ potential. Dealing with three particles makes using the procedure (14) more difficult due to the complexity of particle permutations. For this reason, for the $3 \mathrm{~N}$ case, the symmetrization with respect to discrete transformations is typically divided into two parts as described in [12].

Equation (20) together with the set of operators (19) and table 1 are the final result and can be used to extend the so called "three-dimensional" formalism [7-10] by using more general $2 \mathrm{~N}$ potential types. For the centreof-mass case with $\boldsymbol{K}=0$ our result should reduce to the general form from $[7,13]$ with $\check{w}_{i=1 \ldots 6}\left(\boldsymbol{p}^{\prime}, \boldsymbol{p}\right)$. The resulting relations are

$$
\begin{aligned}
\check{W}_{1}\left(\boldsymbol{p}^{\prime}, \boldsymbol{p}\right)= & \check{W}_{6}\left(\boldsymbol{p}^{\prime}, \boldsymbol{p}, \boldsymbol{K}\right), \\
\check{W}_{2}\left(\boldsymbol{p}^{\prime}, \boldsymbol{p}\right)= & \check{W}_{7}\left(\boldsymbol{p}^{\prime}, \boldsymbol{p}, \boldsymbol{K}\right) \\
\check{W}_{3}\left(\boldsymbol{p}^{\prime}, \boldsymbol{p}\right)= & -\frac{\left(\boldsymbol{p}^{\prime} \times \boldsymbol{p}\right) \cdot\left(\boldsymbol{p}^{\prime} \times \boldsymbol{p}\right) \check{W}_{12}\left(\boldsymbol{p}^{\prime}, \boldsymbol{p}, \boldsymbol{K}\right)}{\boldsymbol{K} \cdot\left(\boldsymbol{p}^{\prime} \times \boldsymbol{p}\right)} \\
& -\frac{\boldsymbol{K} \cdot\left(\left(\boldsymbol{p}^{\prime} \times\left(\boldsymbol{p}^{\prime} \times \boldsymbol{p}\right)\right)+\left(\boldsymbol{p} \times\left(\boldsymbol{p}^{\prime} \times \boldsymbol{p}\right)\right)\right)}{2 \boldsymbol{K} \cdot\left(\boldsymbol{p}^{\prime} \times \boldsymbol{p}\right)} \\
& \times \check{W}_{13}\left(\boldsymbol{p}^{\prime}, \boldsymbol{p}, \boldsymbol{K}\right) \\
& -\frac{\boldsymbol{K} \cdot\left(\left(\boldsymbol{p}^{\prime} \times\left(\boldsymbol{p}^{\prime} \times \boldsymbol{p}\right)\right)-\left(\boldsymbol{p} \times\left(\boldsymbol{p}^{\prime} \times \boldsymbol{p}\right)\right)\right)}{2 \boldsymbol{K} \cdot\left(\boldsymbol{p}^{\prime} \times \boldsymbol{p}\right)} \\
& \times \check{W}_{15}\left(\boldsymbol{p}^{\prime}, \boldsymbol{p}, \boldsymbol{K}\right), \\
\check{w}_{4}\left(\boldsymbol{p}^{\prime}, \boldsymbol{p}\right)= & \frac{1}{2}\left(\boldsymbol{p}^{2}-\boldsymbol{p}^{\prime 2}\right) \check{W}_{2}\left(\boldsymbol{p}^{\prime}, \boldsymbol{p}, \boldsymbol{K}\right) \\
& +\left(\left(\boldsymbol{p}^{\prime} \times \boldsymbol{p}\right) \cdot\left(\boldsymbol{p}^{\prime} \times \boldsymbol{p}\right)\right) \check{W}_{7}\left(\boldsymbol{p}^{\prime}, \boldsymbol{p}, \boldsymbol{K}\right) \\
& +\left(\boldsymbol{p} \cdot \boldsymbol{p}^{\prime}\right) \check{W}_{8}\left(\boldsymbol{p}^{\prime}, \boldsymbol{p}, \boldsymbol{K}^{\prime}\right) \\
& -\frac{1}{2}\left(\boldsymbol{p}^{2}+\boldsymbol{p}^{\prime 2}\right) \check{W}_{9}\left(\boldsymbol{p}^{\prime}, \boldsymbol{p}, \boldsymbol{K}\right), \\
\check{W}_{5}\left(\boldsymbol{p}^{\prime}, \boldsymbol{p}\right)= & \check{W}_{8}\left(\boldsymbol{p}^{\prime}, \boldsymbol{p}, \boldsymbol{K}\right)+\check{W}_{9}\left(\boldsymbol{p}^{\prime}, \boldsymbol{p}, \boldsymbol{K}\right), \\
\check{w}_{6}\left(\boldsymbol{p}^{\prime}, \boldsymbol{p}\right)= & -\check{W}_{8}\left(\boldsymbol{p}^{\prime}, \boldsymbol{p}, \boldsymbol{K}\right)+\check{W}_{9}\left(\boldsymbol{p}^{\prime}, \boldsymbol{p}, \boldsymbol{K}\right) .
\end{aligned}
$$

When taking the limit $\boldsymbol{K} \rightarrow 0$, there is a problem with $\check{w}_{3}$ since some of the scalar functions have singularities. The problem exists only if the scalar functions and operators are considered separately. When taking the limit $\boldsymbol{K} \rightarrow 0$ the product of the scalar function and the operator have to be considered together and the limit can be performed in a straightforward manner. This operation results in the $\breve{w}_{3}$ operator.

\section{Summary}

After introducing a new procedure to generate the rotation-invariant operator form of the $2 \mathrm{~N}$ potential dependent on the spins, relative momenta and totalmomentum operators $\check{\boldsymbol{\sigma}}(1), \check{\boldsymbol{\sigma}}(2), \check{\boldsymbol{p}}, \check{\boldsymbol{p}}^{\prime}, \check{\boldsymbol{K}}$ we added the spatial reflection, particle exchange and time-reversal symmetry. On top of this we required Hermiticity and ended up with the most general form of the $2 \mathrm{~N}$ potential $\check{V}$,

$$
\check{V}=\sum_{i=1}^{16} \beta_{i}^{n_{1}(i), n_{2}(i), n_{3}(i)} \check{W}_{i},
$$

where it is written as a linear combination of scalar functions $\beta^{n_{1}, n_{2}, n_{3}}=\beta^{n_{1}, n_{2}, n_{3}}\left(\boldsymbol{p}, \boldsymbol{p}^{\prime}, \boldsymbol{K}\right)$ and spin operators $\check{W}_{i}=\check{W}_{i}\left(\boldsymbol{p}, \boldsymbol{p}^{\prime}, \boldsymbol{K}\right)$. The spin operators have a known form and the scalar functions (having appropriate properties) effectively define the potential. This final operator form can be used to extend the so called "three-dimensional" formalism by using more general $2 \mathrm{~N}$ potential types. It is a crucial element of these calculations because it significantly reduces the computational complexity of the numerical implementation. Additionally, it can be useful to improve techniques that perform the partial wave decomposition of few nucleon forces. It should be stressed that, with only minor modifications, the techniques described in this paper can also be used to create the general operator form of the $3 \mathrm{~N}$ potential.

We would like to thank Dr. Hermann Krebs from the Ruhr University Bochum for a very insightful email exchange. The project was financed from the resources of the National Science Center (Poland) under grants No. DEC2013/11/N/ST2/03733 and DEC-2013/10/M/ST2/00420.

\section{Appendix A. Illustration of the steps}

Here we give an example that ilustrates the steps in proof from sect. 2. Let us consider the following operator:

$$
\check{X}=(\check{\boldsymbol{p}} \cdot \check{\boldsymbol{\sigma}}(1))(\check{\boldsymbol{p}} \times \check{\boldsymbol{\sigma}}(1) \cdot \check{\boldsymbol{\sigma}}(2))(\check{\boldsymbol{p}} \cdot \check{\boldsymbol{\sigma}}(2)) .
$$

Our aim is to express $\check{X}$ as a linear combination of operators from (7).

We start by looking at the first operator in the chain,

$$
(\check{\boldsymbol{p}} \cdot \check{\boldsymbol{\sigma}}(1)),
$$

and immediately recognize $\check{G}_{4} \in \mathbb{V}_{0}\left(\mathbb{V}_{0}=\mathbb{G}\right)$ from (6). Thus operator (A.1) can be written as

$$
\check{X}=\left(\check{G}_{4}\right)(\check{\boldsymbol{p}} \times \check{\boldsymbol{\sigma}}(1) \cdot \check{\boldsymbol{\sigma}}(2))(\check{\boldsymbol{p}} \cdot \check{\boldsymbol{\sigma}}(2)) .
$$

There are still two additional operators in this chain. This leads us to consider a sub-chain of (A.1) containing the first pair of operators

$$
(\check{\boldsymbol{p}} \cdot \check{\boldsymbol{\sigma}}(1))(\check{\boldsymbol{p}} \times \check{\boldsymbol{\sigma}}(1) \cdot \check{\boldsymbol{\sigma}}(2))
$$

and this time we are looking at $\mathbb{V}_{1}$ since, by construction, any two operator chain can be expressed by operators from $\mathbb{V}_{1}$. It turns out that

$$
(\check{\boldsymbol{p}} \cdot \check{\boldsymbol{\sigma}}(1))(\check{\boldsymbol{p}} \times \check{\boldsymbol{\sigma}}(1) \cdot \check{\boldsymbol{\sigma}}(2))=i \boldsymbol{p}^{2} \check{O}_{8}-i \check{O}_{15},
$$

where $\check{O}_{8}, \check{O}_{15} \in \mathbb{V}_{1}$ are operators from (7). Now we can write (A.1) as

$$
\check{X}=i \boldsymbol{p}^{2} \check{O}_{8}(\check{\boldsymbol{p}} \cdot \check{\boldsymbol{\sigma}}(2))-i \check{O}_{15}(\check{\boldsymbol{p}} \cdot \check{\boldsymbol{\sigma}}(2)) .
$$


Finally, we consider two element sub-chains

$$
\check{O}_{8}(\check{\boldsymbol{p}} \cdot \check{\boldsymbol{\sigma}}(2))
$$

and

$$
\check{O}_{15}(\check{\boldsymbol{p}} \cdot \check{\boldsymbol{\sigma}}(2)) \text {. }
$$

This time we are again looking at $\mathbb{V}_{1}$ since the next iteration $\mathbb{V}_{2}$ does not introduce any new operators. It can easily be checked that

$$
\check{O}_{8}(\check{\boldsymbol{p}} \cdot \check{\boldsymbol{\sigma}}(2))=\check{O}_{4}-i \check{O}_{10}
$$

and

$$
\check{O}_{15}(\check{\boldsymbol{p}} \cdot \check{\boldsymbol{\sigma}}(2))=\boldsymbol{p}^{2} \check{O}_{4} \cdot
$$

Using the above identities we are finally in the position to rewrite (A.1) as

$$
\check{X}=i \boldsymbol{p}^{2}\left(\check{O}_{4}-i \check{O}_{10}\right)-i\left(\boldsymbol{p}^{2} \check{O}_{4}\right)=\boldsymbol{p}^{2} \check{O}_{10} \text {. }
$$

After each step (A.2), (A.3), (A.4) we ended up with shorter chains. Chains from each step start with an operator from $\mathbb{V}_{0}=\mathbb{G}, \mathbb{V}_{1}$ or $\mathbb{V}_{2}=\mathbb{V}_{1}$ respectively.

Open Access This is an open access article distributed under the terms of the Creative Commons Attribution License (http://creativecommons.org/licenses/by/4.0), which permits unrestricted use, distribution, and reproduction in any medium, provided the original work is properly cited.

\section{References}

1. D.R. Entem, R. Machleidt, Phys. Rev. C 68, 041001 (2003).

2. E. Epelbaum, W. Glöckle, Ulf-G. Meißner, Nucl. Phys. A 747, 362 (2005).

3. R. Machleidt, D.R. Entem, Phys. Rep. 503, 1 (2011).

4. E. Epelbaum, M.W. Hammer, Ulf-G. Meißner, Rev. Mod. Phys. 81, 1773 (2009).

5. J.L. Forest, V.R. Pandharipande, J.L. Friar, Phys. Rev. C 52, 568 (1995).

6. P. Vaselý, Lecture notes available at http://www-ucjf . troja.mff.cuni.cz/ ${ }^{\sim}$ vesely/NN_potentials/NN_ potential.pdf.

7. J. Golak, W. Glöckle, R. Skibiński, H. Witała, D. Rozpȩdzik, K. Topolnicki, I. Fachruddin, Ch. Elster, A. Nogga, Phys. Rev. C 81, 043006 (2010).

8. J. Golak, R. Skibiński, H. Witała, K. Topolnicki, W. Glöckle, A. Nogga, H. Kamada, Few-Body Syst. 53, 237 (2012).

9. J. Golak, K. Topolnicki, R. Skibiński, W. Glöckle, H. Kamada, A. Nogga, Few-Body Syst. 54, 2427 (2013).

10. K. Topolnicki, J. Golak, R. Skibiński, H. Witała, C.A. Bertulani, Eur. Phys. J. A 51, 132 (2015).

11. Wolfram Research Inc., Mathematica, Version 10.2 (Champaign, IL, 2015).

12. H. Krebs, A. Gasparyan, E. Epelbaum, Phys. Rev. C 87, 054007 (2013).

13. L. Wolfenstein, Phys. Rev. 96, 1654 (1954). 